\title{
Revisión \\ IMPLICANCIAS DE Campylobacter spp. COMO PATÓGENO ALIMENTARIO
}

\section{Review}

\section{IMPLICATIONS OF Campylobacter spp. AS A FOODBORNE PATHOGEN}

\author{
Gustavo Mardones P. ${ }^{1 *}$, y Juana López M. ${ }^{1}$
}

\author{
${ }^{1}$ Facultad de Ciencias Veterinarias, Dpto. de Patología y Medicina Preventiva, Universidad de \\ Concepción, Casilla 537, Chillán, Chile. \\ * Autor para correspondencia E-mail: gmardones@udec.cl
}

\section{RESUMEN}

Campylobacter spp. es un patógeno alimentario resistente a antibióticos. Es considerado como el primer agente etiológico de diarrea en humanos en los países desarrollados, y el segundo o tercero en países en vías de desarrollo. Las especies termotolerantes de Campylobacter son mundialmente reconocidas por generar la campilobacteriosis, una zoonosis asociada al consumo de alimentos de origen animal. El cuadro clínico generado por estas especies suele ser autolimitado, y la aplicación de un esquema antibiótico solo está recomendado en casos clínicamente severos, debido al desarrollo de patrones de resistencia antibiótica en Campylobacter spp., situación que ha emanado de la incorrecta utilización de estos fármacos en salud animal y humana. A pesar de que las enfermedades transmitidas por alimentos son consideradas de notificación obligatoria, la resistencia antibiótica de Campylobacter spp. no es considerada dentro de la normativa chilena. Por otra parte, su notificación hacia laboratorios de referencia es baja y no está considerado dentro del Reglamento Sanitario de los Alimentos de Chile. El objetivo de esta revisión es describir, en base a publicaciones científicas actualizadas, a Campylobacter spp. como un patógeno alimentario y resistente a antibióticos, estimulando a reconocer al patógeno como un agente de importancia en la industria alimentaria.

Palabras clave: Campylobacter, intoxicación alimentaria, resistencia antibiótica.

\section{ABSTRACT}

Campylobacter spp. is an antimicrobial-resistant foodborne pathogen. It is considered the first etiological agent of diarrhea in humans in developed countries and ranks second or third in developing countries. The thermotolerant Campylobacter species are recognized as a cause of campylobacteriosis, which is a zoonosis associated with the consumption of animal foods. This disease is usually self-limited, and antibiotics should be used to treat severe infections due to the development of antibiotic resistance patterns of Campylobacter spp., which result from the misuse of these drugs in animals or humans. Although foodborne diseases are considered notifiable, antibiotic resistance of Campylobacter spp. is not required by law to be reported in Chile. In fact, its notification to reference laboratories is low, and it is not considered in the Reglamento Sanitario de los Alimentos (Food Sanitary Regulation) of Chile. This review aims at describing Campylobacter spp. as an antibiotic-resistant foodborne pathogen, based on recent scientific publications, also recognizing the importance of this pathogen in the food industry.

Key words: Campylobacter, food poisoning, antibiotic resistance.

Recibido: 13 abril 2016. Aceptado: 16 mayo 2016. 


\section{INTRODUCCIÓN}

El Centro para el Control y Prevención de Enfermedades de EE.UU. (CDC, 2012) indica que las enfermedades transmitidas por alimentos (ETAs) son un problema común en salud pública, costoso, pero prevenible, donde organismos patógenos o sustancias dañinas causantes de enfermedad pueden contaminar los alimentos. Estas enfermedades tienen síntomas diferentes, por lo que no se podría denominar como síndrome (Olea, 2007; CDC, 2012). Campylobacter spp., un bacilo curvo Gram negativo, corresponde a una de las principales causas de infecciones zoonóticas entéricas en el mundo, considerada por la Organización Mundial de la Salud (OMS) como el primer agente etiológico de diarrea en el ser humano en los países desarrollados, y el segundo o tercero en los países en vías de desarrollo, como los de América Latina (Hartnett et al., 2009; FDA, 2012). Por otro lado, Campylobacter coli es la segunda especie más reportada, también implicada en casos de infección alimentaria (Whiley et al., 2013; Hoffmann et al., 2015).

El género Campylobacter contiene 25 especies, siendo C. jejuni la especie de mayor importancia en el hombre; se encuentra como reservorio en el intestino de animales domésticos, principalmente aves de corral, por lo que se puede encontrar en alimentos destinados al consumo humano, tales como carne de pollos broiler y sus subproductos (Kaakoush et al., 2015). C. jejuni se transmite por vía oral, a través de alimentos, y también por contacto con animales infectados (Whiley et al., 2013).

Si bien un inóculo de $10^{4}$ células bacterianas son necesarias para que se produzca la infección, en algunos casos es altamente infectante, provocando la infección con dosis del orden de 500 células (ISP, 2014). La infección gastrointestinal por Campylobacter suele ser autolimitada y leve, con diarrea acuosa, fiebre y dolor abdominal, y solo en el $1 \%$ de los casos deriva en hospitalización (ISP, 2014).

Una de las principales preocupaciones que se presenta con respecto a las especies termotolerantes de Campylobacter, se genera debido al abuso de antibióticos en animales, especialmente en aves de corral destinadas a consumo humano, como también a su uso descontrolado en el tratamiento de la campilobacteriosis en humanos, lo cual promueve el desarrollo de resistencia antibiótica sobre fluoroquinolonas, macrólidos, tetraciclinas, entre otros. Dicha resistencia puede transmitirse por vía alimentaria, convirtiéndose en un problema de salud pública (Epps et al., 2013; CDC, 2014).

Con respecto a la situación nacional, el Regla- mento Sanitario de los Alimentos del Ministerio de Salud de Chile (RSA), establece las condiciones sanitarias que deben cumplir los alimentos, con el fin de asegurar su inocuidad. Este documento señala que los patógenos bacterianos de importancia en la industria de alimentos corresponden a Salmonella spp., Listeria monocytogenes, Escherischia coli, Staphylococcus aureus, Vibrio parahaemolyticus, Clostridium spp. y Bacillus cereus. Sin embargo, Campylobacter spp., un patógeno de importancia alimentaria mundial, no es considerado en este estatuto (MINSAL, 2011). Además, la notificación y derivación de Campylobacter spp. a los laboratorios de referencia es baja, a pesar que desde el año 1983, Campylobacter spp. es agente de vigilancia de laboratorio, según lo establece el Reglamento sobre Notificación de Enfermedades Transmisibles de Declaración Obligatoria (Dto. №158/2004) (Rivera et al., 2011; ISP, 2014). Por otra parte, no es vigilado como un agente afecto a resistencia antibiótica bajo este mismo decreto, a pesar de que existen estudios que señalan que la resistencia antibiótica de este patógeno es una realidad en nuestro país (Pollet et al., 2012; González-Hein et al., 2013; MINSAL, 2014).

Por lo anterior, el objetivo general de esta revisión fue describir y analizar a Campylobacter spp. como un patógeno alimentario y resistente a antibióticos, y los objetivos específicos fueron describir su diversidad patogénica y características como patógeno alimentario, analizar la situación internacional y nacional, analizar la resistencia antibiótica en alimentos y describir métodos de detección en alimentos.

\section{MATERIALES Y MÉTODO}

Para el desarrollo de esta revisión se buscó información en el metabuscador Pubmed, y las bases de datos ScienceDirect, SCOPUS y Web of Science, publicada principalmente entre los años 2008-2015, con la excepción de algunas publicaciones anteriores que son de importancia en el tema. Se usó Google Académico para información obtenida desde páginas de internet, tales como boletines, decretos y normativas.

\section{RESULTADOS Y DISCUSIÓN}

\section{Diversidad patogénica y características de Campylobacter spp.}

El primer reporte de Campylobacter (kampulos, curvo; bacter, bacteria) fue realizado por Theodore Escherich en 1886, quien observó y describió células no cultivables en forma de espiral, pero fue en 1906 identificada por primera vez por dos veterinarios británicos, John McFadyean y Stewart Stockman, quienes reportaron la presen- 
cia de un gran número de un organismo peculiar en el mucus uterino de ovejas preñadas (Epps et al., 2013).

Campylobacter spp., es un género bacteriano perteneciente a la familia Campylobacteraceae. Esta familia fue claramente descrita por P. Vandamme en 1992, quien señaló que también pertenecían a ella los géneros Sulfuruspirillum y Arcobacter. Dentro del género Campylobacter, se han descrito 25 especies bacterianas, correspondientes a $C$. fetus, C. coli, C. concisus, C. curvus, C. gracilis, C. helveticus, C. hominis, C. hyointestinalis, C. jejuni, C. lanienae, C. lari, C. mucosalis, C. rectus, C. showae, C. sputorum, C. upsaliensis, C. insulaenigrae, C. peloridis, C. avium, C. subantarticus, C. canadensis, C. cunicolurum, C. volucris, C. ureoliticus y C. troglodytis, con 8 subespecies (Lapierre, 2013; Kaakoush et al., 2015).

Campylobacter spp. representa un grupo importante de comensales de la mucosa intestinal y bacterias patógenas en humanos y animales. En animales, específicamente en aves, estas especies parecen no causar enfermedad, pero en humanos están asociadas con el desarrollo de patologías (Kaakoush et al., 2015). Dentro de sus características, Campylobacter spp. corresponde a pequeños bacilos no formadores de esporas, de 0,2-0,8 um x 0,5-5 um, con una forma que varía de espiral, a varilla y curvados (Fig. 1), o bien como alas de gaviotas, dependiendo de la especie (Donnison y Ross, 2014). Son móviles gracias a sus flagelos polares, ubicados en uno o los dos extremos de la bacteria (Fig. 1), donde las únicas excepciones son C. gracilis, que no es móvil, y C. showae, que posee múltiples flagelos (Silva et al., 2011). Lapierre (2013) señala que estas estructuras participan en la patogenicidad de la bacteria, gatillado por la capacidad de adherencia e invasión a la célula eucarionte y la producción de citotoxinas.

Con respecto a sus mecanismos de patogenicidad, Campylobacter spp. invade la mucosa intestinal en forma similar a Shigella, llegando a la lámina propia a nivel de intestino delgado y colon, generando una enterocolitis inespecífica, con degeneración y atrofia glandular, pérdida de la producción de mucus, abscesos de las criptas intestinales y ulceración de la mucosa epitelial (ISP, 2014; Bolton, 2015).

En el caso de las citotoxinas, su toxina distensora citoletal (TDC) contribuye a aumentar la patogenicidad de C. jejuni. Este tipo de citotoxinas está ampliamente distribuida en Gram negativos, pero específicamente en Campylobacter spp. causa el arresto celular en fase M/G2 en células eucariontes, evitando que estas desarrollen mitosis y llevándolas a la muerte celular (Bolton, 2015). La TDC está compuesta por 3 sub unidades codificadas por los genes $c d t \mathrm{~A}, c d t \mathrm{~B}$ y $c d t \mathrm{C}$. Se sabe que la sub unidad proteica $\mathrm{CdtB}$, codificada por el gen $c d t \mathrm{~B}$, ingresa a la célula hospedera y se une en la superficie celular con las proteínas CdtA y $\mathrm{CdtC}$, produciendo daño en el ADN celular (Silva et al., 2011; Bolton, 2015). La subunidad que tiene el efecto tóxico es $\mathrm{CdtB}$, la cual, debido a su actividad ADNasa, rompe el ADN y comienza en la célula el mecanismo de reparación que concluye con el arresto celular y la muerte de la célula. Por esto, C. jejuni puede prolongar la persistencia de la enfermedad gastrointestinal, aumentando la inflamación de la mucosa gastrointestinal y el daño hepático. Sin embargo, el efecto citopático puede variar desde un efecto muy importante, a

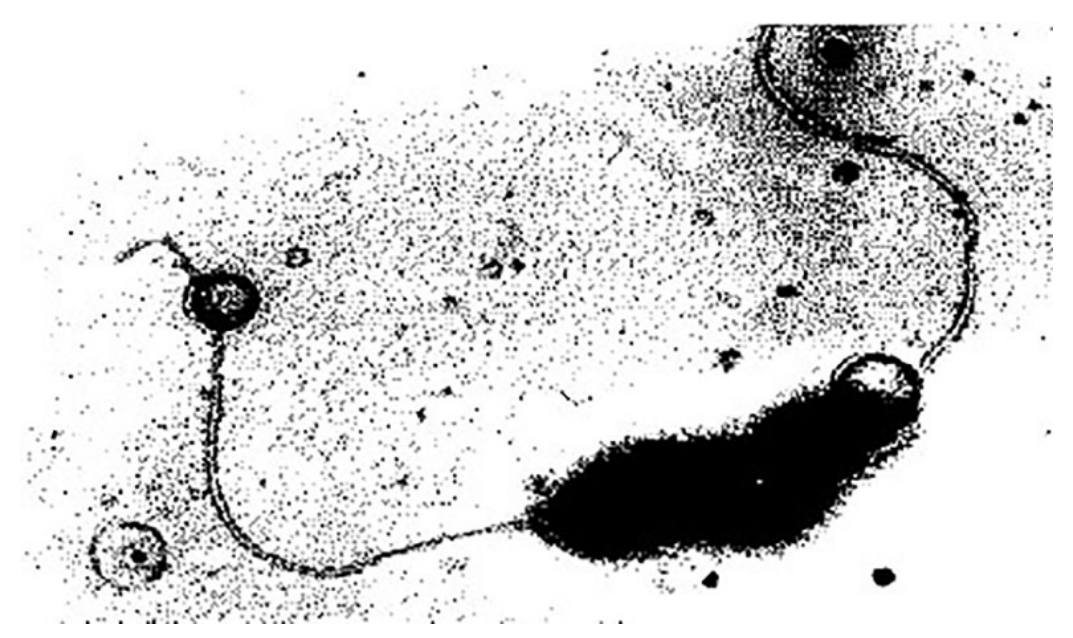

Fig. 1. Microfotografía electrónica mostrando la morfología y la flagelación típicas de Campylobacter (Fernández, 2011b).

Fig. 1. Electron micrograph showing typical morphology and flagellation of Campylobacter. (Fernández, 2011b). 
estar ausente in vivo. Igualmente, la TDC es altamente prevalente en Campylobacter spp. (Lapierre, 2013).

Campylobacter spp. se encuentra ampliamente distribuido en la naturaleza. Suelo, estiércol, ambientes acuáticos y agua son los nichos naturales donde se suele encontrar, y desde aquí es capaz de generar infección en el humano (Whiley et al., 2013). Una gran variedad de animales domésticos y de vida silvestre son su reservorio natural, tales como ganado vacuno, cerdos, ovejas, aves de corral, cabras, perros, gatos y roedores, incluso en monos (Silva et al., 2011; Fernández y Oval, 2013; Koga et al., 2014).

La dosis infectante de Campylobacter es de $10^{4}$ microorganismos, los que se multiplican en el intestino delgado, destruyen la mucosa intestinal, invaden el epitelio y producen inflamación con infiltración de leucocitos en la lámina propia, con presencia de leucocitos en las heces en el 25-80\% de los casos (Silva et al., 2011). Elementos estructurales de C. jejuni como el flagelo, proteínas de superficie y lipopolisacáridos de superficie (LPS) le otorgan la capacidad de actuar como adhesina, y puede adherirse a las células epiteliales y al moco intestinal. Además, la forma curva o espiral y la atracción quimiotáctica del moco intestinal hacia la bacteria, facilita su contacto con el epitelio (Fernández, 2011b; Silva et al., 2011).

Campylobacter spp. posee la capacidad de formar biopelículas en superficies inertes, tales como vidrio, acero inoxidable y plástico, así como también en agua y tejidos del hospedador, con el fin de asegurar su sobrevivencia, resistiendo estrés físico, biológico y medioambiental. Películas en el aire o en líquidos, cúmulos agregados a superficies o flóculos desagregados, son las formas de persistencia y diseminación que utiliza el biofilm para resistir condiciones adversas, con flóculos bacterianos que pueden sobrevivir por sobre 24 días a temperatura ambiental. Las biopelículas también protegen a las bacterias residentes de antibióticos, defensas del huésped y bacteriófagos inmunes (Lapierre, 2013; Kaakoush et al., 2015).

El cuadro clínico desarrollado por la infección con Campylobacter spp. es la campilobacteriosis, enfermedad de baja mortalidad y distribución mundial, que afecta a individuos de todas las razas, sexos y edades. Consiste en una enteritis que cursa con diarrea acuosa o sanguinolenta, nauseas, fiebre y dolor abdominal, con un cuadro autolimitado, que no suele durar más de 6 días (FDA, 2012; Whiley et al., 2013). Sin embargo, se ha reportado que $1 \%$ de los casos requieren hospitalización por complicaciones y con secuelas post infecciosas, afectando principalmente al sistema nervioso, como es el caso del síndrome de Guillain Barré, polineuropatía inflamatoria desmielinizante, producto de una reacción autoinmune de los anticuerpos contra Campylobacter spp., que dañan el tejido nervioso periférico. En otros casos puede existir artritis reactiva e incluso aborto (Kaakoush et al., 2015).

La mayoría de los casos reportados de campilobacteriosis se deben a las especies termotolerantes, principalmente $C$. jejuni, C. coli y $C$. lari, las cuales debido a su capacidad de crecer a $42^{\circ} \mathrm{C}$, pueden invadir el organismo de animales de sangre caliente, inclusive humanos, y causar enfermedad, generando un cuadro clínico similar entre las diversas especies, pero difiriendo en su reservorio principal (Tabla 1) (Fernández, 2011b; FDA, 2012; Kaakoush et al., 2015). Wagenaar et al. (2014) señalan que sobre un $90 \%$ de los casos de campilobacteriosis son causados por $C$. jejuni o C. coli, mientras que un pequeño número se debe a C. fetus. Sin embargo, el cuadro inducido por C. coli es detectado con menor frecuencia que $C$. jejuni en muchas zonas del planeta; mientras que en países desarrollados es responsable de 3-5\% de los casos de diarrea producidos por Campylobacter termotolerante, en países en desarrollo puede llegar a 25\% (FDA, 2012; Donnison y Ross, 2014).

C. jejuni, la causa más frecuente de infección en el hombre, comprende dos subespecies; $C$. jejuni subesp. jejuni y C. jejuni subesp. Doylei. La principal diferencia entre ellas radica en que $C$. jejuni subesp. jejuni crece mejor entre 37 y $42^{\circ} \mathrm{C}$, la temperatura corporal aproximada de las aves $\left(41^{\circ} \mathrm{C}\right.$ a $\left.42^{\circ} \mathrm{C}\right)$, y parece estar bien adaptada a esta especie, pues son las principales portadoras de la enfermedad (OIE, 2008; Fernández, 2011b). En

Tabla 1. Especies y subespecies de Campylobacter y su principal reservorio animal. Table 1. Campylobacter species and subspecies and its main animal reservoir.

\begin{tabular}{ll}
\hline Especie/Subespecie & Reservorio principal \\
\hline C. jejuni subesp. doylei & Aves y mamíferos \\
C. jejuni subesp. jejuni & Humanos, perros y gallinas \\
C. coli & Cerdos \\
C. lari & Aves marinas (gaviotas) \\
\hline
\end{tabular}

Modificado de Fernández, 2011b. 
contraste con Salmonella, no puede crecer fuera de un hospedador de sangre caliente, debido a la ausencia de un ambiente microaerofílico y a las temperaturas poco adecuadas para multiplicarse, sin embargo, puede sobrevivir en el medio ambiente, según la especie de que se trate y condiciones medioambientales, tales como temperatura, luz, interacciones bióticas, concentración de oxígeno y nutrientes (EFSA, 2011). Se ha logrado determinar que en condiciones de estrés ambiental, como una reducción de $\mathrm{pH}$ en el procesamiento o almacenamiento de alimentos, puede adquirir una forma cocoide, que corresponde a formas viables pero no cultivables de la célula vegetativa (VNC) que le permiten sobrevivir en condiciones desfavorables por largos periodos de tiempo. Con respecto a la temperatura, al no poseer proteínas para el shock por frio, son incapaces de crecer bajo $30^{\circ} \mathrm{C}$, mientras que crecen óptimamente entre un $\mathrm{pH}$ de 6,5-7,5, lo que le permite subsistir en carne y leche (Silva et al., 2011; Bolton, 2015).

Otras especies como C. concisus, C. lari y C. ureolyticus han adquirido importancia porque han incrementado su presencia tanto en humanos como en animales (Fernández, 2011b).

Campylobacter spp. en alimentos de origen animal. Se piensa que la principal ruta de transmisión de Campylobacter spp. termotolerantes corresponde a carnes y subproductos cárnicos crudos. La microbiota intestinal y carne de las aves de corral, especialmente de pollos broiler, constituyen uno de los principales reservorios y fuente de infección de Campylobacter en el hombre (Rivera et al., 2011; Zendehbad et al., 2015). Gran cantidad de pollos en los planteles avícolas resultan infectados con Campylobacter spp., ya sea por hábitos como la coprofagia, contaminación de alimentos y agua con las heces, o por contacto fecal-oral entre los individuos, no presentando signos clínicos, y pasando desapercibidos en los controles sanitarios tradicionales. Posteriormente, cuando el ave infectada es llevada a matadero, el microorganismo puede pasar desde los intestinos a la musculatura, en el proceso de la evisceración, contaminando la carne (Silva et al., 2011; Riley et al., 2015; Zendehbad et al., 2015). Sin embargo, en un estudio para determinar el nivel de contaminación en canales de pollos broiler con C. jejuni, realizado por Gruntar et al. (2015), se detectó una mayor contaminación en la fase posterior al desplume, lo que sugiere que la contaminación más importante se produce antes de la entrada a la sala de evisceración, debido a la corta duración de la fase de escaldado $\left(\right.$ a $54^{\circ} \mathrm{C}$ ) y la consecuente sobrevivencia de especies de Campylobacter en la canal, posterior al desplume. Aunque todas las especies de aves de consumo pueden contener Campylobacter spp., el riesgo de transmisión alimentaria es mayor en el pollo, debido a las grandes cantidades comercializadas por la industria avícola (CDC, 2014; Marotta et al., 2015). Debido a que el bovino también es un reservorio de Campylobacter spp., la carne es considerada un alimento afecto a la contaminación con este patógeno, ya sea directamente o por contaminación ambiental (Fernández y Hitschfeld, 2009).

En el caso de la industria lechera, la leche puede contaminarse con este patógeno a través de la ubre, o bien en la extracción, por tanto la leche no pasteurizada representa un posible vehículo de transmisión de Campylobacter spp. (Fernández y Hitschfeld, 2009; Rivera et al., 2011).

Campylobacter spp. en aguas. Las aguas superficiales y cauces de agua pueden estar contaminadas por la presencia de heces infectadas de vacunos o aves, transmitiendo el patógeno a otros animales o incluso a personas que consuman estas aguas contaminadas (Whiley et al., 2013).

Campylobacter spp. en vegetales. Se ha podido determinar la presencia de $C$. jejuni en vegetales almacenados a $4^{\circ} \mathrm{C}$, especialmente verduras de hoja verde, tales como lechugas (Lactusa sativa), espinacas (Spinacia oleracea) y perejil (Petroselinum crispum), asociándose la intoxicación al consumo de vegetales crudos (Guévremont et al., 2015). Esto afirmaría la teoría que Campylobacter spp. puede sobrevivir a temperatura de refrigeración en diferentes medioambientes biológicos por largos periodos, por medio de respuestas adaptativas al frio (Duffy y Dykes, 2006; Guévremont et al., 2015).

Por lo anterior, la aplicación de buenas prácticas de higiene en la granja y de manufactura de alimentos, tanto en industria como en el hogar, son necesarias para evitar la presencia de Campylobacter spp. en la cadena alimentaria.

Prevalencia de Campyobacter spp. a nivel internacional. Este género es reconocido como los mayores patógenos bacterianos desde mediados de 1990 en el humano, donde los métodos de detección perfeccionados permitieron su registro en Europa y Norte América (Whiley et al., 2013); C. jejuni es la especie que mayor cantidad de casos genera dentro del género (Kaakoush et al., 2015). Tanto en EE.UU. como en Inglaterra, Campylobacter se aísla con mayor frecuencia que Salmonella y Shigella, mientras que en Dinamarca constituye la causa más común de enfermedad zoonótica transmitida por alimentos (Gilliss et al., 2013; Rossi et al., 2015). Whiley et al. (2013) aseveran que cerca de un $80 \%$ de los casos de Campylobacter en EE.UU. en un año típico corresponden a 
ETAs, por tanto, los alimentos constituyen una de las principales fuentes de infección. Se estima que afecta a 1,3 millones de personas cada año en EE.UU., con un incremento de $14 \%$ en la incidencia de campilobacteriosis en el 2012 con respecto a los años 2006-2008, superando a otros patógenos alimentarios, tales como Cryptosporidium, Listeria, Salmonella, Shigella y E. coli O157:H7 Shiga toxigénica (Gilliss et al., 2013; Rossi et al., 2015).

Los datos de Thomas et al. (2013) reportan una incidencia de 145.000 casos en los 32,5 millones de habitantes en Canadá, lo que ubica a $C$. jejuni como el tercer agente patógeno alimentario de mayor importancia en este país, luego de norovirus y Clostridium perfringens.

En la Unión Europea, el número de casos confirmados de campilobacteriosis el año 2012 fueron 214.000 , con un promedio de 55 casos por 100.000 . Little et al. (2010) señalan que se reportaron 50000 casos de campilobacteriosis en Inglaterra y Gales durante el año 2008, habiendo una tendencia al alza para el año 2009 , con un $17 \%$ más de casos que el año anterior. En Alemania, 70.560 casos fueron reportados el 2011, lo que significó una prevalencia mayor que Salmonella, Shigella, Yersinia y Listeria (Kaakoush et al., 2015) Por otro lado, en el 2010 fue la ETA más frecuentemente notificada en Australia, con 16.968 casos en todos los estados (OzFoodNetWorkingGroup, 2012).

Los estudios realizados en países asiáticos arrojan resultados similares al resto del mundo. En China, la investigación de la etiología de gastroenteritis en tres hospitales de Yangzhou entre el 2005 y 2006 muestran que 14,9\% de los pacientes con gastroenteritis fueron positivos a Campylobacter, con 4,84\% de 3.061 pacientes con diarrea positivos a $C$. jejuni, en un hospital en Beijing en un estudio entre 2005-2009. (Huang et al., 2009; Chen et al., 2011).

En India, un estudio en un hospital de Calcuta, indica que en el periodo entre enero del 2008 y diciembre del 2010, 7\% de los hospitalizados por gastroenteritis fueron positivos a especies de Campylobacter, con un 70\% de los aislados que identificaron a C. jejuni como agente causal. En esta región de India, la campilobacteriosis es más prevalente en niños bajo 5 años (Mukherjee et al., 2013). Los estudios avalan que los infantes presentan la mayor cantidad de aislados positivos a $C$. jejuni y $C$. coli, siendo endémicos en la población pediátrica, y que adultos jóvenes de sexo masculino también presentan alta cantidad de aislados positivos. Finalmente, los estudios indican que la campilobacteriosis ocurre en las zonas cálidas con mayor frecuencia durante los meses de verano (Mason et al., 2013; Rossi et al., 2015; Zendehbad et al., 2015).

En América Latina, las especies termotoleran- tes de Campylobacter son agentes importantes de diarrea, pero se encuentran en un porcentaje menor que E. coli enteropatógeno y Shigella, que son predominantes en estas zonas, donde la transmisión a través del agua y el contacto directo con animales infectados son las principales vías de infección en seres humanos (Fernández, 2011a; ISP, 2014). Fernández (2011a) señala que un hallazgo frecuente en esta región del mundo es la presencia de portadores sanos y alto grado de deshidratación de los enfermos, lo que no ocurre en países industrializados, asociado a bajas condiciones de saneamiento básico y bajo estatus nutricional, transmitiéndose especies diarreogénicas de Campylobacter a niños de corta edad. Otra situación que denotan los investigadores, es que $C$. jejuni y C. coli son la principal causa de diarrea de los viajeros. La CDC señala que aproximadamente un 19\% de los casos de Campylobacter spp. identificados en FoodNet están asociados con viajeros de países desarrollados en países en desarrollo (CDC, 2014; Zendehbad et al., 2015).

Prevalencia de Campylobacter spp. a nivel nacional. En Chile, debido a que la mayoría de los laboratorios no está preocupado de estudiar este agente, sumado a la falta de implementación de técnicas diagnósticas, la notificación y derivación de Campylobacter spp. hacia los laboratorios de referencia es baja. Esto a pesar que desde el año 1983, Campylobacter spp. es agente de vigilancia de laboratorio según lo establece el reglamento sobre notificación de enfermedades transmisibles de declaración obligatoria. Por lo demás, no está incluido dentro del RSA chileno, por tanto no existen márgenes regulatorios precisos sobre los límites en los que Campylobacter spp. resulta nocivo al ser ingerido en alimentos (MINSAL, 2011).

Estudios realizados en Chile demuestran una alta prevalencia de Campylobacter spp. en los alimentos. En un estudio en mataderos nacionales, se determinó un $72 \%$ de prevalencia en canales de pollos broiler en 2 de 4 de los mataderos estudiados, posterior a la etapa de evisceración (Figueroa et al., 2009). Por otra parte, en un estudio para determinar la prevalencia de Campylobacter spp. en el territorio nacional, el ISP (2014) confirmó 462 muestras positivas, en un periodo comprendido entre enero del 2005 y agosto del 2013, con mayor cantidad de cepas positivas el 2011. El detalle de este estudio señala que un $93,5 \%$ de las cepas confirmadas provenían de la Región Metropolitana, mientras que solo un 1,5\% provenía de Arica y Parinacota; $1,5 \%$ del Biobío y 1,3\% del Libertador B. O'Higgins, con mayor prevalencia en el género masculino y en niños de 1-9 años, dominando ampliamente $C$. jejuni por sobre $C$. coli y C. fetus (ISP, 2014). 


\section{Antecedentes sobre resistencia antibiótica de Campylobacter spp.}

La resistencia antibiótica de Campylobacter spp. es considerada por la OMS como un serio problema en salud pública (Rossi et al., 2015). La administración de concentraciones bajo la dosis bactericida a poblaciones susceptibles, puede conllevar a mutaciones en la especie bacteriana, desarrollando la resistencia a determinado antibiótico, donde la dinámica de la resistencia a antimicrobianos es altamente dependiente de la clase de antibiótico, concentraciones administradas y mutaciones relacionadas con cada agente antimicrobiano. Esto debido al surgimiento de variadas especies de Campylobacter zoonótico a partir de animales domésticos, y de la transmisión del patógeno resistente a alimentos de consumo humano. A raíz de esta situación, se desencadena una mayor duración de la enfermedad, riesgo de enfermedad invasiva y muerte, así como aumento de los costos en el cuidado de la salud (Bolton, 2015; Kaakoush et al., 2015; Rossi et al., 2015).

El desarrollo de resistencia antibiótica se genera principalmente en el intestino de las especies animales que son reservorio para Campylobacter spp., sobre todo en aquellas que son blanco del uso desmedido de antibióticos. Cepas resistentes son capaces de transmitirse fácilmente a través del medio ambiente, por ejemplo, vía plantas de tratamiento de aguas residuales de desechos humanos y animales, transmitiéndose también fácilmente entre especies, por ejemplo entre aves domésticas, aves silvestres y el ser humano. En este sentido y por razones desconocidas, cepas de C. coli aisladas desde aves de consumo y otros animales, parecen tener una multirresistencia antibiótica más importante incluso que cepas de $C$. jejuni (Rossi et al., 2015).

Por lo anterior, es esencial abordar el gran dilema de aplicar un tratamiento antibiótico en individuos enfermos, considerando que los antibióticos solo están recomendados en casos graves o en individuos inmunocomprometidos, donde existan síntomas tales como fiebre, diarrea sanguinolenta, bacteremia, inmunocompromiso, riesgo de infección en embarazo, presencia de más de 8 episodios diarreicos en $24 \mathrm{~h}$, o bien, persistencia de síntomas por más de 7 días (FDA, 2012; Whiley et al., 2013; Kaakoush et al., 2015). A raíz de esto, Rossi et al (2015) especifican que países desarrollados como EE.UU., Suiza, Australia, Finlandia y otros países del norte de Europa, controlan estrictamente el uso de antibióticos en medicina veterinaria, por tanto poseen actualmente bajos niveles de resistencia antibiótica. Sin embargo, considerando pacientes que han realizado viajes internacionales, es posible la propagación de patrones de resistencia hacia sectores desarrollados.
Con respecto a los mecanismos utilizados por Campylobacter para la adquisición de resistencia antibiótica, estos son similares al resto de las bacterias Gram negativas. Los antibióticos comúnmente asociados con la adquisición de resistencia antibiótica son las fluoroquinolonas, macrólidos, tetraciclinas, aminoglicósidos y betalactámicos, donde el esquema general para tratar a los pacientes con campilobacteriosis corresponde a eritromicina (macrólido) en niños y ciprofloxacino (fluoroquinolona) en adultos (Fig. 2) (FDA, 2012; Kaakoush et al., 2015; Rossi et al., 2015). Alteraciones del sitio de unión del antibiótico al patógeno, por medio de la expresión de genes que alteran dicho sitio; bomba de eflujo CmeABC, para estimular la salida del fármaco desde la célula bacteriana; enzimas que contrarrestan o anulan al antibiótico y su efecto; y alteración de la permeabilidad de membrana por la expresión de porinas de membrana externa (MOMP), son los principales mecanismos de resistencia bacteriana a estos antibióticos (Fig. 2). (Rivera et al., 2007; García et al., 2009; Kim et al., 2010; Pollet et al., 2012; Iovine, 2013; Kaakoush et al., 2015; Rossi et al., 2015). Debido a que estos mecanismos muchas veces no son antibiótico específicos, es que se ha desarrollado además una multirresistencia de especies termotolerantes de Campylobacter hacia estos antimicrobianos (González-Hein et al., 2013; Bolton, 2015; Zendehbad et al., 2015).

Es por todo lo anterior que Campylobacter es vigilado constantemente en la Unión Europea, EE.UU. y Canadá, para poder planificar métodos para combatir la resistencia antibiótica de Campylobacter spp. (ISP, 2014; Rossi et al., 2015). En Chile aún existen muy pocos estudios que analicen la resistencia antibiótica en Campylobacter spp., por lo que se hace necesario considerarlo como una problemática mayor para la salud pública nacional. Preocupantemente, fluoroquinolonas y macrólidos están autorizados de acuerdo al registro de medicamentos veterinarios del Servicio Agrícola y Ganadero, y también se utilizan en medicina humana. Se sabe también que la cantidad de antibióticos del grupo de las fluoroquinolonas destinados a uso veterinario es muy superior al que se usa en medicina humana (Rivera et al., 2007; García et al., 2009; Kaakoush et al., 2015). Es por esto que es necesario incentivar prácticas que reduzcan el desarrollo de resistencia antibiótica en salud animal y humana, evitando el uso profiláctico y como promotor de crecimiento de antibióticos e integrar a las especies termotolerantes de Campylobacter a vigilancia como agentes resistentes a antimicrobianos en el Dto. № 158/2004, para mejorar el control de Campylobacter resistente a antibióticos en alimentos (MINSAL, 2014). 


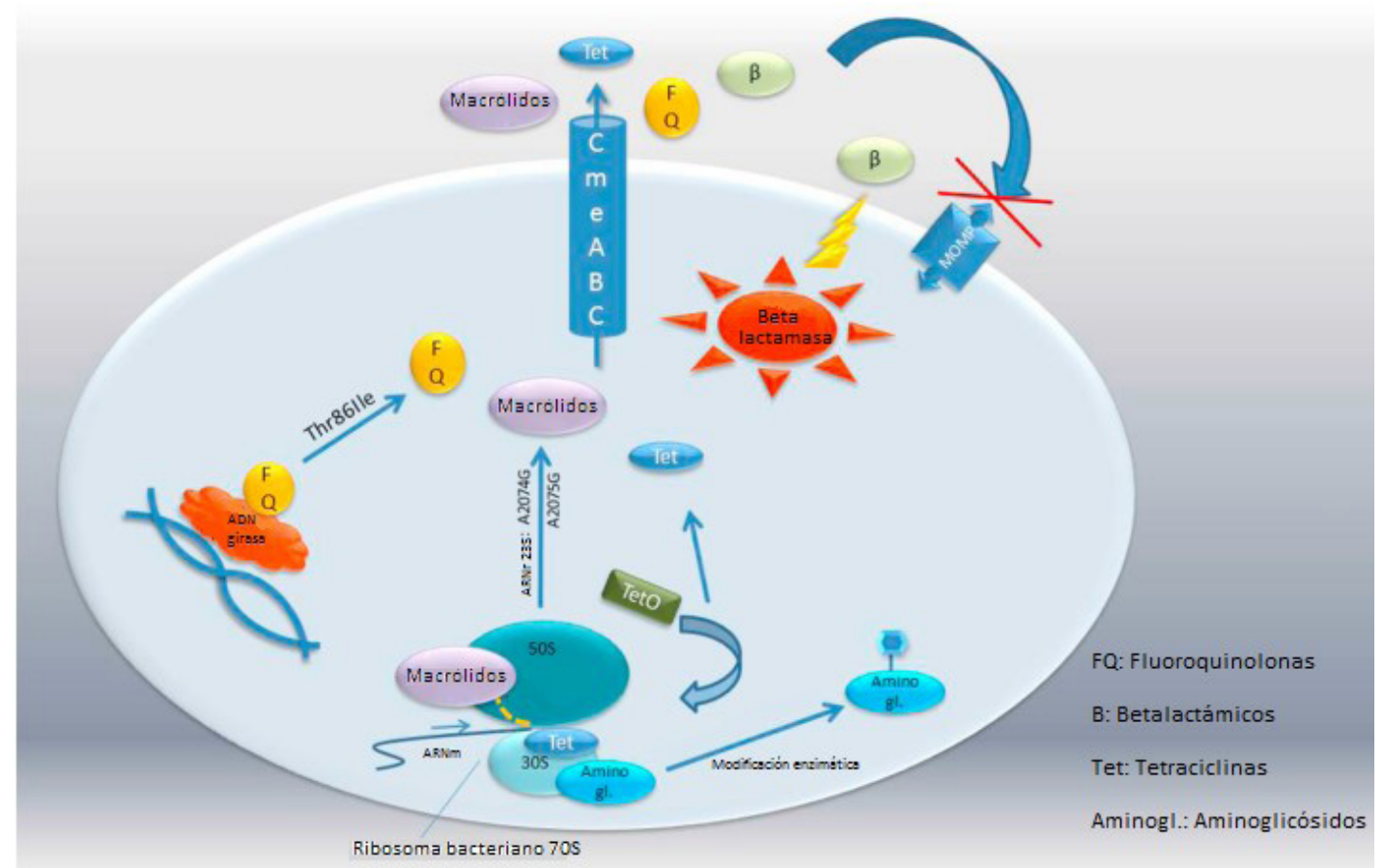

Fig. 2. Esquema que representa los blancos celulares de la actividad antimicrobiana y los mecanismos de resistencia antibiótica (Modificado de Rossi et al., 2015).

Fig. 2. Scheme representing the cellular targets of antimicrobial activity and mechanisms of antibiotic resistance (Modificado de Rossi et al., 2015).

Métodos de detección de Campylobacter spp. Campylobacter spp. no es fermentador de carbohidratos y se nutre de aminoácidos. Por tanto, los medios para su detección deben ser ricos en nutrientes aminoacídicos, con una mezcla antibiótica que inhiba a contaminantes fecales y permita la selección de Campylobacter spp. (FDA, 2012; CDC, 2014). La detección de las especies termotolerantes de Campylobacter es compleja, y requiere de varios métodos combinados para lograr sensibilidad y especificidad. Whiley et al. (2013) señalan que son 3 los métodos de identificación clásicos: el cultivo tradicional por medio de agar selectivo, filtración por membrana en el agar sangre, y métodos moleculares de detección.

Cultivo, filtración por membrana y métodos confirmativos. El protocolo recomendado por la ISO 10272 (2006) para Campylobacter spp. señala que se necesita llevar la bacteria a caldo selectivo para luego sembrar en agar de cultivo selectivo. Con respecto a ello, el caldo Preston, o bien, el caldo Bolton, son medios selectivos de enriquecimiento de las especies termotolerantes de Campylobacter spp. que afectan alimentos (Habib et al., 2011). Luego, la muestra debe ser llevada a cultivo en agar selectivo Skirrow, donde las colo- nias positivas toman una coloración rosado pálido, aspecto redondeadas, con un borde regular, convexo, liso y brillante. Por otra parte, el agar selectivo desoxicolato-cefaperazona-carbon modificado (agar $\mathrm{mCCD}$ ), recomendado por la ISO 10272 (2006) genera colonias grisáceas, planas y húmedas, con tendencia a expandirse, y un brillo metálico, en muchos casos (OIE, 2008; Habib et al., 2011). Ya que se produce el crecimiento de flora acompañante, el método de filtración por membrana, el cual filtra las muestras a través de una membrana de celulosa triacetato, separa a Campylobacter spp. de otras especies bacterianas de rápido crecimiento, que puedan desarrollarse en el agar. El problema en este medio, es que la bacteria tiende a entrar en un estado viable no cultivable (VNC) (OIE, 2008; Habib et al., 2011; Whiley et al., 2013).

Para la confirmación, pruebas como motilidad positiva, catalasa y oxidasa positiva, crecimiento microaeróbico a $25^{\circ} \mathrm{C}$, o crecimiento aeróbico a $42^{\circ} \mathrm{C}$, permiten confirmar a especies termotolerantes de Campylobacter. La hidrolisis de hipurato permite diferenciar a C. jejuni del resto de las especies termotolerantes (Tabla 2) (OIE, 2008; Habib et al., 2011; Donnison y Ross, 2014). 
Tabla 2. Reacciones bioquímicas típicas de las tres especies mayormente aisladas de Campylobacter termotolerante (Modificado de Donnison y Ross, 2014).

Table 2. Typical biochemical reactions of the three mainly isolated thermotolerant Campylobacter species (Modificado de Donnison y Ross, 2014).

\begin{tabular}{lccc}
\hline Característica & C. jejuni & C. coli & C. lari \\
\hline Crecimiento a $25^{\circ} \mathrm{C}$ & - & - & - \\
Crecimiento a $42^{\circ} \mathrm{C}$ & + & + & + \\
Catalasa & + & + & + \\
Hidrolisis de hipurato & + & - & - \\
\hline
\end{tabular}

Métodos moleculares. El PCR en tiempo real, es una técnica altamente sensible que amplifica el ADN bacteriano e indica instantáneamente la cepa bacteriana, ayuda a delinear a nivel de especie en Campylobacter, identificando además estados VNC, con resultados en un día. Sin embargo, puede conllevar a una sobre estimación del recuento, debido a la amplificación en estado VNC o muerto, pues se ha demostrado que el ADN bacteriano es muy estable, y puede persistir por 14 días en sedimento marino y agua salada. Es por ello que para mejorar la detección de células vivas intactas, se utiliza la técnica de etidio monoácido (EMA) en conjunto con el PCR en tiempo real. EMA se une al ADN que no está protegido por una membrana celular y así evita la amplificación y enumeración de células amorfas, mejorando la eficacia de las técnicas moleculares (OIE, 2008; Whiley et al., 2013).

\section{CONCLUSIONES}

El género Campylobacter está compuesto por 25 especies bacterianas. C. jejuni y C. lari son las especies que mayor cantidad de casos generan en el mundo, causando una enterocolitis de transmisión alimentaria. Alimentos como carne de pollo y leche no pasteurizada se relacionan con casos ETAs por Campylobacter spp.

Campylobacter spp. es considerado como el primer agente etiológico de diarrea en países desarrollados, y el segundo o tercero en países en vías de desarrollo. En Chile, su notificación hacia los laboratorios de referencias es baja, y no está incluida en el RSA chileno.

Debido al abuso de antibióticos en salud animal y humana, Campylobacter spp. ha desarrollado resistencia hacia antibióticos de primera elección en el tratamiento de la campilobacteriosis.

Finalmente, la combinación de cultivo bacteriológico, método de filtración por membrana y métodos moleculares resulta la técnica más efectiva para su detección en alimentos.

\section{LITERATURA CITADA}

Bolton, D.J. 2015. Campylobacter virulence and survival factors. Food Microbiol. 48:99-108.

CDC. 2012. Food safety. Center for Disease Control and Prevention (CDC). Available at http://www.cdc.gov/foodsafety/facts.htm1\#what (Accessed 5 June 2015).

CDC. 2014. Campylobacter. Center for Disease Control and Prevention (CDC). Available at http://www.cdc.gov/nczved/divisions/ $\mathrm{dfbmd} /$ diseases/campylobacter/ (Accessed 5 June 2015).

Chen, J., X.T. Sun, Z. Zeng, and Y.Y. Yu. 2011. Campylobacter enteritis in adult patients with acute diarrhea from 2005 to 2009 in Beijing, China. Chin. Med. J. 124:1508-1512.

Donnison, A.M., and C.M. Ross. 2014. Thermotolerant Campylobacter. p. 382-388. In M. Dickeman and C. Devine (eds.). Encyclopedia of Meat Sciences. 2nd. ed. Volume 2. Academic Press, Jamestown Road, London, UK.

Duffy, L., and G.A. Dykes. 2006. Growth temperature of four Campylobacter jejuni strains influences their subsequent survival in food and water. Lett. App. Microbiol. 43:596-601.

Epps, S.V.R., R.B. Harvey, M.E. Hume, T.D. Phillips, R.C. Anderson, and D.J. Nisbet. 2013. Foodborne Campylobacter: infections, metabolism, pathogenesis and reservoirs. Int. J. Environ. Res. Public Health. 10:6292-6304.

EFSA. 2011. Scientific opinion on Campylobacter in broiler meat production: control options and performance objectives and/or targets at different stages of the food chain. European Food Safety Authority. EFSA Journal 9:8-75.

Fernández, H. 2011a. Campylobacter y campilobacteriosis: una mirada desde América del Sur. Rev. Peru Med. Exp. 28:121-27.

Fernández, H. 2011b. Campylobacter: un género de bacterias zoonóticas de interés en salud pública. Rev. Med. Maule. 28:52-59. 
Fernández, H., and M. Hitschfeld. 2009. Occurrence of Campylobacter jejuni and Campylobacter coli and their biotypes in beef and dairy cattle from the south of Chile. Bra. J. Microbiol. 40:450-454.

Fernández, H., and A. Oval. 2013. Occurrence of Campylobacter jejuni and Campylobacter coli biotypes and antimicrobial susceptibility in healthy dogs in southern Chile. Acta Sci. Vet. 41:1-5.

Figueroa, G., M. Troncoso, C. López, P. Rivas, and M. Toro. 2009. Occurrence and enumeration of Campylobacter spp. during the processing of Chilean broilers. BMC Microbiology 9:1-6.

FDA. 2012. BBB-Campylobacter jejuni. Food and Drug Administration (FDA). Available at http://www.fda.gov/food/foodborneillnesscontaminants/causesofillnessbadbugbook/ ucm070024.htm (Accessed 5 June 2015).

García, P., N. Valenzuela, V. Rodríguez, E. León, y H. Fernández. 2009. Susceptibilidad antimicrobiana de Campylobacter jejuni aislado de coprocultivos en Santiago de Chile. Rev. Chil. Infect. 26:511-514.

Gilliss, D., A.B. Cronquist, M. Cartter, M. Tobin-D'Angelo, D. Blythe, and K. Smith. 2013. Incidence and trends of infection with pathogens transmitted commonly through food. Foodborne Diseases Active Surveillance Network, 10 U.S. sites, 1996-2012. Morbidity and Mortality Weekly Report 62(15):283-287.

González-Hein, G., N. Cordero, P. García, y G. Figueroa. 2013. Análisis molecular de la resistencia a fluoroquinolonas y macrólidos en aislados de Campylobacter jejuni de humanos, bovinos y carne de ave. Rev. Chi. Infect. 30:135-139.

Gruntar, I., M. Biasizzo, D. Kusar, M. Pate, and M. Ocepek. 2015. Campylobacter jejuni contamination of broiler carcasses: Population dynamics and genetic profiles at slaughterhouse level. Food Microbiol. 50:97-101.

Guévremont, E., L. Lamoureux, P. Ward, and S. Villeneuve. 2015. Survival of Campylobacter jejuni on fresh spinach stored at $4^{\circ} \mathrm{C}$ or $12^{\circ} \mathrm{C}$. Food Control 50:736-739.

Habib, I., M. Uyttendaele, and L. De Zutter. 2011. Evaluation of ISO 10272:2006 standard versus alternative enrichment and plating combinations for enumeration and detection of Campylobacter in chicken meat. Food Microbiol. 28:1117-1123.

Hartnett, E., A. Fazil, G. Paoli, M. Nauta, B. Bak Christensen, H. Rosenquist, et al. 2009. Evaluación de riesgos de Campylobacter spp. en pollos para asar. Resumen interpretativo. $p$. 19-49. Serie de evaluación de riesgos microbiológicos $\mathrm{N}^{\circ} 11$. FAO/WHO, Roma, Italia.
Hoffmann, S., B. Maculloch, and M. Batz. 2015. Economic burden of major foodborne illnesses acquired in the United States. United State Departament of Agriculture, Washington D.C., USA. Bulletin/EIB 140:18-19.

Huang, J.L., H.Y. Xu, G.Y. Bao, X.H. Zhou, D.J. Ji, and G. Zhang. 2009. Epidemiological surveillance of Campylobacter jejuni in chicken, dairy cattle and diarrhea patients. Epidemiol. Infect. 137:1111-1120.

ISP. 2014. Vigilancia de laboratorio de Campylobacter spp. Chile, 2005-2013. Instituto de Salud Pública (ISP), Ministerio de Salud, Santiago, Chile. Boletín ISP 4(1):1-17.

Iovine, N.M. 2013. Resistance mechanisms in Campylobacter jejuni. Virulence 4:230-240.

Kaakoush, N.O., H.M. Mitchell, and S.M. Man. 2015. Campylobacter. p. 1187-1222. In Y. Tang, M. Sussman, D. Li, I. Poxton, and J. Schwartzman (eds.) Molecular Medical Microbiology. Vol. 1. 2nd. ed.. Academic Press, Jamestown Road, London, UK.

Kim, J.M., J. Hong, W. Bae, H.C. Koo, S.H. Kim, and Y.H. Park. 2010. Prevalence, antibiograms, and transferable tet $(\mathrm{O})$ plasmid of Campylobacter jejuni and Campylobacter coli isolated from raw chicken, pork, and human clinical cases in Korea. J. Food Protect. 73:1430-1437.

Koga, T., W. Aoki, T. Mizuno, K. Wakazono, J. Ohno, T. Nakai, et al. 2014. Antimicrobial resistance in Campylobacter coli and Campylobacter jejuni in cynomolgus monkeys (Macaca fascicularis) and eradication regimens. J. Microbiol. Immunol. 50:75-82.

Lapierre, L. 2013. Factores de virulencia asociados a especies zoonóticas de Campylobacter spp. Av. Cienc. Vet. 28:25-31.

Little, C.L., F.J. Gormley, N. Rawal, and J.F. Richardson. 2010. A recipe for disaster: outbreaks of campylobacteriosis associated with poultry liver pate in England and Wales. Epidemiol. Infect. 138:1691-1694.

Marotta, F., G. Garofolo, G. Di Donato, G. Aprea, I. Platone, S. Cianciavicchia, et al. 2015. Population diversity of Campylobacter jejuni in poultry and its dynamic of contamination in chicken meat. Biomed Res. Int. DOI 10.1155/2015/859845.

Mason, J., M. Iturriza-Gomara, S.J. O'Brien, B.M. Ngwira, W. Dove, and M.C. Maiden. 2013. Campylobacter infection in children in Malawi is common and is frequently associated with enteric virus coinfections. PLOS ONE 8:e59663. 
MINSAL. 2011. Reglamento Sanitario de los Alimentos. Ministerio de Salud (MINSAL), Chile. Disponible en http://www.sernac.cl/ wpcontent/ uploads/2012/11/ reglamento-sanitario-alimentos-2011.pdf (Consulta 5 junio 2015).

MINSAL. 2014. Reglamento sobre notificación de enfermedades transmisibles de declaración obligatoria. D.S. N 158/2004. Ministerio de Salud, Santiago, Chile.

Mukherjee, P., T. Ramamurthy, M.K. Bhattacharya, K. Rajendran and A.K. Mukhopadhyay. 2013. Campylobacter jejuni in hospitalized patients with diarrhea, Kolkata, India. Emerg. Infect. Dis. 19:1155-1156.

Olea, A.M. 2007. Las enfermedades transmitidas por alimentos: un fenómeno frecuente de magnitud real desconocida. El Vigía. 25:3742.

OIE. 2008. Manual de la OIE sobre animales terrestres. Capítulo 2.9.3. Campylobacter jejuni y Campylobacter coli. Organización Mundial de Sanidad Animal (OIE). Disponible en http://web.oie.int/esp/normes/mmanual/ pdf_es_2008/2.09.03.\%20Campilobacter\%20 jejuni.pdf (Consulta 5 junio 2015).

Pollett, S., C. Rocha, R. Zerpa, L. Patiño, A. Valencia, M. Camiña, et al. 2012. Campylobacter antimicrobial resistance in Perú: a ten-year observational study. BMC Infect. Dis. DOI: 10.1186/1471-2334-12-193.

Riley, A., A. Eshaghi, R. Olsha, V.G. Allen, and S.N. Patel. 2015. Antibiotic susceptibility of clinical isolates of Campylobacter jejuni and Campylobacter coli in Ontario, Canada during 2011-2013. Diagn. Mic. Infec. Dis. 83(3):292294.

Rivera, N., H. Fernández, R. Bustos, M.E. Valenzuela, N. Trabal, S. Montenegro, et al. 2007. Susceptibilidad antimicrobiana de cepas de Campylobacter aisladas de carcasas de aves, sangre y fecas humanas. Rev. Latinoam. Actual. Biomed. 1:32-37.
Rivera, N., R. Bustos, S. Montenegro, M. Sandoval, J. Castillo, H. Fernández, et al. 2011. Genotipificación y resistencia antibacteriana de cepas de Campylobacter spp. aisladas en niños y en aves de corral. Rev. Chil. Infectol. 28:555-562.

Rossi, M., S. Olkkola, M. Roasto, R. Kivistö, and M.L. Hänninen. 2015. Antimicrobial resistance and Campylobacter jejuni and C. coli. p. 55-69. In C. Chen, X. Yan and C.R. Jackson (eds.) Antimicrobial resistance and food safety. Academic Press, London, UK.

Silva, J., D. Leite, M. Fernandes, C. Mena, P.A. Gibbs, and P. Teixeira. 2011. Campylobacter spp. as a foodborne pathogen: a review. Front. In Microbiol. 2:1-12. DOI: 10.3389/ Fmicb.2011.00200.

OzFoodNet Working Group. 2012. Monitoring the incidence and causes of diseases potentially transmitted by food in Australia: annual report of the OzFoodNet network. Commun. Dis. Intell. Q. Rep. 34:396-426.

Thomas, M.K., R. Murray, L. Flockhart, K. Pintar, F. Pollari, A. Fazil, et al. 2013. Estimates of the burden of foodborne illness in Canada for 30 specified pathogens and unspecified agents, circa 2006. Foodborne Pathog. Dis. 10(7):639-648.

Wagenaar, J.A., M.A.P. van Bergen, M.J. Blaser, R.V. Tauxe, D.G. Newell, and J.P.M. van Putten. 2014. Campylobacter fetus infections in humans: exposure and disease. Clin. Infect. Dis. 58:1579-1586.

Whiley, H., B. van den Akker, S. Giglio, and R. Bentham. 2013. The role of environmental reservoirs in human campylobacteriosis. Int. J. Environ. Res. Public Health 10:5886-5907.

Zendehbad, B., J. Khayatzadeh, and A. Alipour. 2015. Prevalence, seasonality and antibiotic susceptibility of Campylobacter spp. isolates of retail broiler meat in Iran. Food Control 53:41-45. 